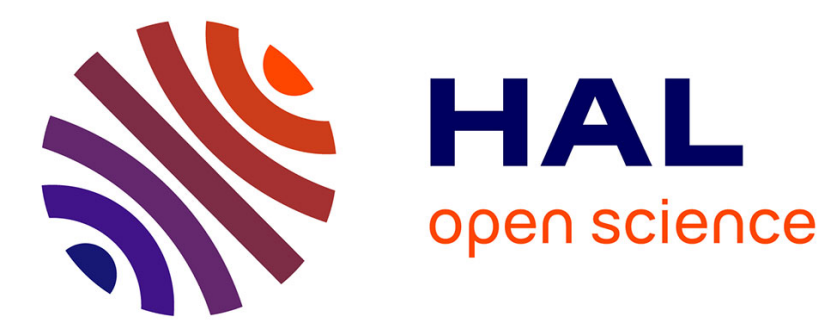

\title{
Stability Analysis of Systems with Delay-Dependant Coefficients a two-Parameter Approach
}

Chi Jin, Keqin Gu, Islam Boussaada, Silviu-Iulian Niculescu

\section{To cite this version:}

Chi Jin, Keqin Gu, Islam Boussaada, Silviu-Iulian Niculescu. Stability Analysis of Systems with Delay-Dependant Coefficients a two-Parameter Approach. ACC 2017 - American Control Conference, May 2017, Seattle, WA, United States. 10.23919/acc.2017.7963847 . hal-02276317

\section{HAL Id: hal-02276317 \\ https://hal-centralesupelec.archives-ouvertes.fr/hal-02276317}

Submitted on 7 Jul 2020

HAL is a multi-disciplinary open access archive for the deposit and dissemination of scientific research documents, whether they are published or not. The documents may come from teaching and research institutions in France or abroad, or from public or private research centers.
L'archive ouverte pluridisciplinaire HAL, est destinée au dépôt et à la diffusion de documents scientifiques de niveau recherche, publiés ou non, émanant des établissements d'enseignement et de recherche français ou étrangers, des laboratoires publics ou privés. 


\title{
Stability Analysis of Systems with Delay-Dependant Coefficients: a two-Parameter Approach
}

\author{
Chi Jin ${ }^{1}$, Keqin $\mathrm{Gu}^{2}$, Islam Boussaada ${ }^{1}$, Silviu-Iulian Niculescu ${ }^{3}$,
}

\begin{abstract}
Stability of systems with a single delay and delaydependent coefficients is studied along the line of the $\tau$ decomposition approach. Criteria for determining crossing directions of imaginary characteristic roots with possibly multiplicity are presented, with which system stability for any given delay value can be determined in a systematic way. In contrast to the previous research on this type of systems, our analysis is based on a novel two-parameter framework. With the new geometric insight, stronger criteria concerning the crossing direction of imaginary characteristic roots with possibly multiplicity can be obtained using simplified and intuitive arguments. The stability analysis procedure is illustrated with an example inspired by biological applications.
\end{abstract}

\section{INTRODUCTION}

Time-delay systems appear in a wide range of applications including population dynamics, network control and biological systems, and have attracted significant attention from the mathematical, control and biological community. See the books [3], [5], [6] for summaries of the progress made in this field.

A number of effective methods have been proposed for linear delay systems with delay-free coefficients in [1], [2], [13]. These methods are along the line of D-decomposition [14], [15] and are based on the fact that the solutions of the characteristic equation depend on the system parameters continuously. As a special case, the so-called $\tau$-decomposition method [16] regards the time delay as the parameter. The general idea of this method is first to identify the critical delay values for which the system admit imaginary roots. Those critical delay values separate the delay interval into several subintervals. By identifying the crossing direction of these imaginary roots, one can determine the change of the number of characteristic roots on the right half plane as $\tau$ sweeps through each critical delay, and thus determine system stability for any delay value.

However, in some practical applications the system coefficients may depend on the delay. These models include the stellar dynamos [11], the hematopoietic stem cell dynamics [12], and the population models with age structure [9].

\footnotetext{
1 Chi Jin and Islam Boussaada are with IPSA \& Laboratoire des Signaux et Systèmes (L2S) CentraleSupélec-CNRS-Université Paris Sud, 3 rue Joliot-Curie 91192 Gif-sur-Yvette cedex, France. (chi. jin, islam.boussaada) (al2s. centralesupelec.fr

${ }^{2}$ Keqin Gu is with Department of Mechanical and Industrial Engineering, Southern Illinois University Edwardsville, Edwardsville, Illinois 62026, USA. kguasiue.edu

3 Silviu-Iulian Niculescu is with Laboratoire des Signaux et Systèmes (L2S) CentraleSupélec-CNRS-Université Paris Sud, 3 rue Joliot-Curie 91192 Gif-sur-Yvette cedex, France. Silviu.NICULESCU@l2s. centralesupelec.fr
}

The characteristic equation of this population model can be written as

$$
\lambda^{2}+a \lambda+c+(b(\tau) \lambda+d(\tau)) e^{-\lambda \tau}=0
$$

where $a=m_{J}+m_{A}+a_{J} s, b(\tau)=\left(a_{A}-a_{J}\right) s e^{-m_{J} \tau}$, $c=m_{A} m_{J}+a_{J} m_{A} s, d(\tau)=\left(a_{A} m_{J}-a_{J} m_{A}\right) s e^{-m_{J} \tau}$.

Beretta and Kuang studied a restricted class of such systems and proposed an effective method of stability analysis based on the graph of functions [9]. Our previous work [17] relaxed some restrictive assumptions in [9] and developed a more complete method for stability analysis.

However, the method developed in [17] is still restrictive in the sense that no higher-order derivatives have been obtained, and numerical method may have to be used if the locus of a characteristic root near the imaginary axis is tangent to the imaginary axis as the delay varies. Another restriction is that it cannot treat multiple imaginary roots. In this paper, we develop a two-parameter approach. We first view the delay parameter in coefficients and in the state as two independent parameters, say $r$ and $q$, respectively. Then stability is first investigated on the $r-q$ plane and the restraint $r=q=\tau$ is subsequently imposed to determine the stability of the original system. This method is more geometrical in nature, and it is able to remove the restrictions of the method in [18]. Specifically, it can treat systems with multiple imaginary characteristic roots, or locus of characteristic roots tangent to the imaginary axis. When appropriate differentiability conditions are satisfied, higher-order derivatives may also be derived based on this formulation, thus allowing us to derive crossing directions analytically in more general cases.

The following notation will be used in this paper. Let $\operatorname{sgn}(\cdot)$ denote the sign function. For any complex number $s, \mathfrak{R}(s)$ notes the real part of $s$. We use $\mathscr{B}_{\delta}(s)$ to denote a closed ball with radius $\delta>0$ centred at some point $s$ on the complex plan.

\section{Problem Statement}

We consider systems with the following characteristic equation:

$$
D(\lambda, \tau)=P(\lambda, \tau)+Q(\lambda, \tau) e^{-\lambda \tau}=0,
$$

where $P(\lambda, \tau)$ and $Q(\lambda, \tau)$ are polynomials in $\lambda$ and continuous in $\tau$. Function $P(\lambda, \tau), Q(\lambda, \tau), D(\lambda, \tau)$ may be written as $P_{\tau}(\lambda), Q_{\tau}(\lambda)$ and $D_{\tau}(\lambda)$, respectively, to emphasize that they are considered as functions of $\lambda$ with $\tau$ as the parameter. Introduce a function $F$ as

$$
F(\omega, \tau)=P(j \omega, \tau) P(-j \omega, \tau)-Q(j \omega, \tau) Q(-j \omega, \tau) .
$$


Clearly if $\lambda=j \omega$ is an imaginary characteristic root of $D_{\tau}(\lambda)$, we must have

$$
F(\omega, \tau)=0
$$

We may also denote $F(\omega, \tau)$ as $F_{\tau}(\omega)$. The same $F$ function is also introduced in [9] and [17].

We now state a set of standing assumptions that hold throughout this paper.

Assumption I. For all $\tau \in \mathscr{I}$, there exits $n \geq 0$ such that the order of polynomial $P_{\tau}$ equals $n$. The following also holds for $\tau \in \mathscr{I}$ :

$$
\lim _{\omega \rightarrow \infty}\left|\frac{Q_{\tau}(j \omega)}{P_{\tau}(j \omega)}\right|<1
$$

Assumption II. No $(\omega, \tau) \in \mathbb{R}_{+} \times \mathscr{I}$ satisfies

$$
\left\{\begin{array}{l}
P(j \omega, \tau)=0 \\
Q(j \omega, \tau)=0
\end{array}\right.
$$

simultaneously.

Assumption III. There are only a finite number of pairs $(\omega, \tau)$ in $\mathbb{R}_{+} \times \mathscr{I}$ that simultaneously satisfy (4) as well as

$$
\partial_{\omega} F(\omega, \tau)=0
$$

Define $\mathscr{T}_{F}$ as the set of the $\tau$ that appear in such pairs.

Assumption IV. For all $\tau \in \mathscr{I}, \lambda=0$ is not a characteristic root of $D_{\tau}(\lambda)$. Furthermore, let $\mathscr{T}_{c}$ be the set of all $\tau \in \mathscr{I}$ for which $D_{\tau}(\lambda)$ admits imaginary roots. Each element of $\mathscr{T}_{c}$ is referred to as a critical delay. Any bounded delay interval contains at most a finite number of critical delays.

Assumption I-IV should hold for general systems with characteristic equations of the form (2) except for some degenerated cases. See [17] for more detailed discussion of this aspect as well as the implication of these assumptions. In our previous work [9], we require $P(\lambda, \tau)$ and $Q(\lambda, \tau)$ to be differentiable at any critical delay. It is also assume there that if $j \omega^{*}$ is an imaginary root of $D_{\tau^{*}}(\lambda)$, then $F_{\tau^{*}}^{\prime}\left(\omega^{*}\right) \neq 0$. These assumptions are no longer required in this work.

Our objective is to determine the delay intervals contained in $\mathscr{I}$ for which system (2) is asymptotically stable. For this purpose, We will also investigate how imaginary roots migrate as $\tau$ increases and sweeps through the critical delay.

\section{STABILITY ANALYSIS}

The main idea for stability analysis here is along the line of the $\tau$-decomposition method. We arrange the elements of $\mathscr{T}_{c}$, namely all the critical delays, in an ascending order as

$$
\tau^{l} \leq \tau_{1}<\tau_{2}<\cdots<\tau_{L} \leq \tau^{u} .
$$

Our assumptions guarantee that there exists $c>0$ such that any characteristic root of $D_{\tau}(\lambda)$ with real part greater than $-c$ varies continuously with $\tau$ [17]. Consequently, as $\tau$ sweeps through $\mathscr{I}$, the number of characteristic roots on the right half complex plane (R.H.P) is constant in any interval that contains no critical delay.

There are two important aspects of the stability analysis: i) Identifying the values of $\tau$ such that there is at least one root of $D_{\tau}(\lambda)$ on the imaginary axis, as well as the corresponding imaginary roots. ii) Determining how imaginary roots migrate, namely whether these imaginary roots move from the R.H.P to the left half complex plane (L.H.P), or vise versa, or merely touches the imaginary axis and then returns to the original side as $\tau$ increases through these values.

In this section, we will consider the first aspect and provide a precise formulation of the root migration problem. Criteria for computing the crossing direction of the imaginary roots will be given in the next section.

\section{A. Conditions for imaginary characteristic roots}

Recall the set $\mathscr{T}_{F}$ defined in Assumption III. We agree to order the elements of $\mathscr{T}_{F} \cup\left\{\tau^{l}, \tau^{u}\right\}$ in an ascending order

$$
\tau^{(0)}<\tau^{(1)}<\cdots<\tau^{K}
$$

Then, we may partition $\mathscr{I}$ into $K$ subintervals:

$$
\mathscr{I}^{(i)}=\left[\tau^{(i-1)}, \tau^{(i)}\right], i=1,2, \ldots, K .
$$

It has been shown in [17] that the number of real roots of $F(\omega, \tau)$ in $\omega$ are constant for all $\tau$ in the interior of $\mathscr{I}^{(i)}$, and they are all simple. These roots are continuous functions of $\tau$ in $\mathscr{I}^{(i)}$ and denoted as $\omega_{k}^{(i)}(\tau), k=1,2, \cdots, m(i)$, and $m(i)$ is the number of real roots of $F_{\tau}(\omega)$ for $\tau \in \mathscr{I}^{(i)}$. We refer to the graph of each $\omega_{k}^{(i)}(\tau)$ as a frequency curve. We further introduce a set $\Omega_{F}(\tau)$ that collects all different values of the real roots of $F_{\tau}(\omega)$. More precisely, we define:

$$
\Omega_{F}(\tau)=\bigcup_{k=1,2, \cdots, m(i)}\left\{\omega_{k}^{(i)}(\tau)\right\} \text { if } \tau \in \mathscr{I}^{(i)}
$$

Since $\partial_{\omega} F\left(\omega_{k}^{(i)}(\tau), \tau\right) \neq 0$ for any $\tau$ in the interior of $\mathscr{I}^{(i)}$, we can denote:

$$
\operatorname{sgn}_{k}^{(i)}=\operatorname{sgn}\left(\partial_{\omega} F\left(\omega_{k}^{(i)}(r), r\right)\right), \forall r \in\left(\tau^{(i-1)}, \tau^{(i)}\right) .
$$

Then $\operatorname{sgn}_{k}^{(i)}$ is just a constant number. For given $i, k$, define:

$$
\begin{aligned}
\theta_{k}^{(i)}(\tau)= & \angle P\left(j \omega_{k}^{(i)}(\tau), \tau\right)-\angle Q\left(j \omega_{k}^{(i)}(\tau), \tau\right) \\
& +\omega_{k}^{(i)}(\tau) \tau+\pi .
\end{aligned}
$$

Here the quantity $\angle P\left(j \omega_{k}^{(i)}(\tau), \tau\right)$ measures the phase angle of $P\left(j \omega_{k}^{(i)}(\tau), \tau\right)$ and is continuous on $\mathscr{I}^{(i)}$. Similar properties also apply to the quantity $\angle Q\left(j \omega_{k}^{(i)}(\tau), \tau\right)$. These two quantities are well defined in view of Assumption II. Consequently, each function $\theta_{k}^{(i)}(\tau)$ is continuous on $\mathscr{I}^{(i)}$ and its range is not limited to any interval of $2 \pi$. The graph of each $\theta_{k}^{(i)}(\tau)$ is referred to as a phase curve.

It is easy to see that $j \omega^{*}$ is an imaginary characteristic root of $D_{\tau^{*}}(\lambda), \tau^{*} \in \mathscr{I}$ if and only if there exist some $i, k$ such that

$$
\theta_{k}^{(i)}(\tau)=2 l \pi, l \text { integer }
$$

and $\omega^{*}= \pm \omega_{k}^{(i)}\left(\tau^{*}\right)$. Therefore one can obtain $\mathscr{T}_{c}$, the set of all critical delays by solving (12). 
Regarding the population model (1), we set $m_{A}=0.2$, $m_{J}=0.1, s=1, a_{J}=0.5, a_{A}=3$ and analyse the system for $\tau \in \mathscr{I}=\left[\tau^{l}, \tau^{u}\right]=[0,14]$. We have:

$$
\begin{aligned}
P(\lambda, \tau) & =\lambda^{2}+a \lambda+c, Q(\lambda, \tau)=b(\tau) \lambda+d(\tau) \\
F(\omega, \tau) & =\left(c-\omega^{2}\right)^{2}+|a \omega|^{2}-|b(\tau) \omega|^{2}-d(\tau)^{2} \\
& =\omega^{4}+\omega^{2}\left(a^{2}-b^{2}(\tau)-2 c\right)+c^{2}-d^{2}(\tau)
\end{aligned}
$$

Using (13), (6) can be written as

$$
\partial_{\omega} F(\omega, \tau)=4 \omega^{3}-2 \omega\left(a^{2}-b^{2}(\tau)-2 c\right) .
$$

Solving (4) together with (6) for $(\omega, \tau) \in \mathbb{R} \times \mathscr{I}$ we obtain two solutions in $\tau$, namely $\tau \approx 6.108$ and $\tau \approx 11.695$. By definition $\tau^{(0)}=0, \tau^{(1)} \approx 6.108$ and $\tau^{(2)} \approx 11.695$. Therefore $\mathscr{I}$ can be decomposed into $\mathscr{I}^{(1)}=\left[0, \tau^{(1)}\right], \mathscr{I}^{(2)}=\left[\tau^{(1)}, \tau^{(2)}\right]$, $\mathscr{I}^{(3)}=\left[\tau^{(2)}, \tau^{u}\right]$. From (13) we further obtain

$$
\begin{aligned}
& \omega_{1}^{(1)}(\tau)=2^{-\frac{1}{2}} \sqrt{b^{2}(\tau)+2 c-a^{2}+\Delta^{\frac{1}{2}}(\tau)}, \tau \in \mathscr{I}^{(1)} \\
& \omega_{1}^{(2)}(\tau)=2^{-\frac{1}{2}} \sqrt{b^{2}(\tau)+2 c-a^{2}+\Delta^{\frac{1}{2}}(\tau)}, \tau \in \mathscr{I}^{(2)} \\
& \omega_{2}^{(2)}(\tau)=2^{-\frac{1}{2}} \sqrt{b^{2}(\tau)+2 c-a^{2}-\Delta^{\frac{1}{2}}(\tau)}, \tau \in \mathscr{I}^{(2)}
\end{aligned}
$$

where $\Delta(\tau)=\left(b^{2}(\tau)+2 c-a^{2}\right)^{2}-4\left(c^{2}-d^{2}(\tau)\right)$. The graph of $\omega_{1}^{(1)}(\tau), \omega_{1}^{(2)}(\tau), \omega_{2}^{(2)}(\tau)$ are plotted in the upper diagram of Fig.1. We have function $\theta_{1}^{(1)}(\tau)$ defined in $\mathscr{I}^{(1)}$ and functions $\theta_{1}^{(2)}(\tau), \theta_{2}^{(2)}(\tau)$ defined in $\mathscr{I}^{(2)}$. The graph of these functions are plotted in the lower diagram of Fig. 1. We find the graph of $\theta_{1}^{(1)}(\tau)$ intersects the horizontal line located at 0 at point $\mathrm{D}$ and the corresponding delay value is $\tau_{1} \approx 0.846$. The graph of $\theta_{2}^{(2)}(\tau)$ intersects the horizontal line located at $2 \pi$ at point $\mathrm{E}$ and the corresponding delay is $\tau_{2}=11.566$. By definition $\mathscr{T}_{c}=\left\{\tau_{1}, \tau_{2}\right\}$.
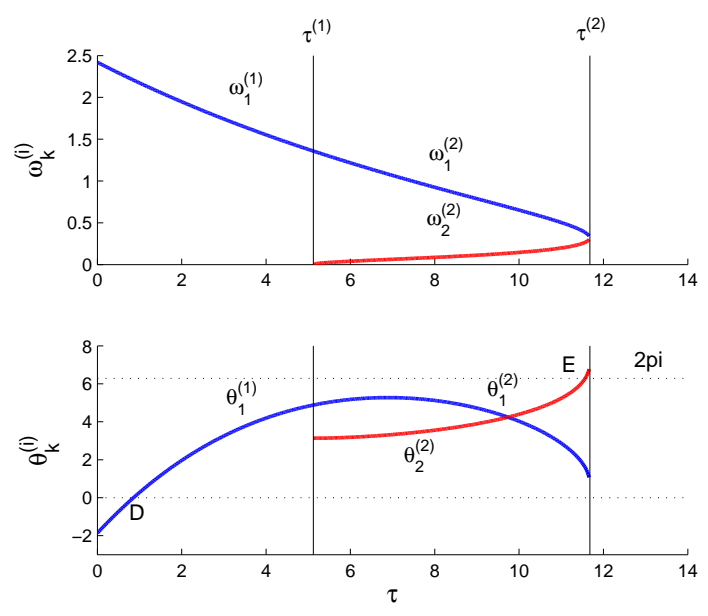

Fig. 1. The graph of function $\omega_{k}^{(i)}$ and $\theta_{k}^{(i)}$ associated with the population model (1).

\section{B. Counting unstable roots}

Suppose $j \omega^{*}$ is an imaginary characteristic root of $D_{\tau^{*}}(\lambda)$ with multiplicity $\mu \geq 1$ for some critical delay $\tau^{*} \in \mathscr{I}$. The continuous dependence of the characteristic roots on $\tau$ means that for any sufficiently small positive number $\delta$, one can find $\varepsilon(\delta)>0$ such that for any $\Delta \tau$ with an absolute value smaller than $\varepsilon(\delta), D_{\tau^{*}+\Delta \tau}(\lambda)$ has exactly $\mu$ roots within $\mathscr{B}_{\delta}\left(j \omega^{*}\right)$. We will investigate how these imaginary roots migrate as $\tau$ sweeps through a small neighbourhood of $\tau^{*}$. To make this problem precise, we define $N^{u}\left(\tau, \mathscr{B}_{\delta}\left(j \omega^{*}\right)\right)$ as the number of roots of $D_{\tau}(\lambda)$ contained in $\mathscr{B}_{\delta}\left(j \omega^{*}\right) \cap$ R.H.P. Recall the set $\mathscr{T}_{c}$ introduced in Assumption IV and the set $\Omega_{F}(\tau)$ defined in (9). For each $\tau^{*} \in \mathscr{T}_{c}$ and $\omega^{*} \in \Omega_{F}\left(\tau^{*}\right)$ define:

$$
\begin{aligned}
\operatorname{Inc}\left(\omega^{*}, \tau^{*}\right)= & \frac{1}{2} \lim _{\varepsilon \rightarrow 0^{+}}\left(N^{u}\left(\tau^{*}+\varepsilon, \mathscr{B}_{\delta}\left(j \omega^{*}\right)\right)\right. \\
& \left.-N^{u}\left(\tau^{*}-\varepsilon, \mathscr{B}_{\delta}\left(j \omega^{*}\right)\right)\right), \text { if } \tau^{*} \neq \tau^{l}, \\
\operatorname{Inc}\left(\omega^{*}, \tau^{*}\right)= & \lim _{\varepsilon \rightarrow 0^{+}} N^{u}\left(\tau^{*}+\varepsilon, \mathscr{B}_{\delta}\left(j \omega^{*}\right)\right) \\
& -N^{u}\left(\tau^{*}, \mathscr{B}_{\delta}\left(j \omega^{*}\right)\right), \text { if } \tau^{*}=\tau^{l} \\
\operatorname{Inc}\left(\tau^{*}\right)= & \sum_{\omega^{*} \in \Omega_{F}\left(\tau^{*}\right)} 2 \operatorname{Inc}\left(\omega^{*}, \tau^{*}\right) .
\end{aligned}
$$

As $\tau$ sweeps increasingly through a critical delay value $\tau^{*}$, the quantity $\operatorname{Inc}\left(\omega^{*}, \tau^{*}\right)$ counts how many roots migrate toward R.H.P through the point $j \omega^{*}$ and the quantity $\operatorname{Inc}\left(\tau^{*}\right)$ is just equal to the change of unstable characteristic roots. The coefficient 2 in (16) is due to the symmetry of imaginary roots about the real axis. If $\lambda=j \omega^{*}$ is a simple characteristic root, then $\operatorname{Inc}\left(\omega^{*}, \tau^{*}\right)=1$ means this root crosses the imaginary axis towards R.H.P. It moves towards L.H.P if $\operatorname{Inc}\left(\omega^{*}, \tau^{*}\right)=-1$. Otherwise this root merely touches the imaginary axis but does not cross it.

We will refer to the characteristic roots on R.H.P as the unstable roots. We shall determine the number of unstable roots for any given $\tau \in \mathscr{I}$ denoted as $N^{u}(\tau)$. It is easy to see the following relation holds for $\tau \in \mathscr{I}-\mathscr{T}_{c}$ :

$$
N^{u}(\tau)=N^{u}\left(\tau^{l}\right)+\sum_{k=1}^{L(\tau)} \operatorname{Inc}\left(\tau_{k}\right)
$$

where $L(\tau)$ is the index of the largest critical delay that is smaller than $\tau$, or equivalently $\tau_{L(\tau)}<\tau$.

\section{CRossing CONDITIONS}

In this section we will provide formula to compute the quantity $\operatorname{Inc}\left(\omega^{*}, \tau^{*}\right)$ introduced in the last section. For this purpose, we will first present a novel two-parameter perspective of systems with delay-dependent coefficients to gain some geometric insight.

\section{A. A two-parameter perspective}

Consider the characteristic equation

$$
\tilde{D}(\lambda, r, q)=P(\lambda, r)+Q(\lambda, r) e^{-\lambda q}=0,
$$


where $q, r \in \mathscr{I}$ are two independent parameters. Then equation (2) becomes equivalent to (18) if we impose the restriction $q=r=\tau$. We denote

$$
\tilde{D}_{r q}(\lambda)=\tilde{D}(\lambda, r, q)
$$

For any $r \in \mathscr{I}^{(i)}$ that satisfies $\omega_{k}^{(i)}(r) \neq 0$, define

$$
\tau_{k}^{(i)}(r)=\frac{\angle Q\left(j \omega_{k}^{(i)}(\tau), \tau\right)-\angle P\left(j \omega_{k}^{(i)}(\tau), \tau\right)-\pi}{\omega_{k}^{(i)}(r)} .
$$

In (18) let Parameter $r \in \mathscr{I}$ be fixed. It is easy to see that a necessary and sufficient condition for $j \omega^{*}, \omega^{*}>0$ to be a characteristic root of $\tilde{D}_{r q^{*}}(\lambda)$ is that the following equations

$$
\begin{gathered}
\omega^{*}=\omega_{k}^{(i)}(r), \\
q^{*}=\tau_{k}^{(i)}(r)+2 l \pi / \omega_{k}^{(i)}(r), l \text { integer, }
\end{gathered}
$$

hold together for some $i, k$.

Consider the square region $\mathscr{I} \times \mathscr{I}$ on the $r-q$ parameter plane. Our analysis so far has shown that as the parameter point $(r, q)$ moves in this region, (18) admits some imaginary roots if and only if $(r, q)$ lies on one of the critical delay curves. Therefore the critical delay curves split the parameter domain on the $r-q$ plane into closed sub-regions and within the interior of each sub-region the number of unstable roots is invariant.

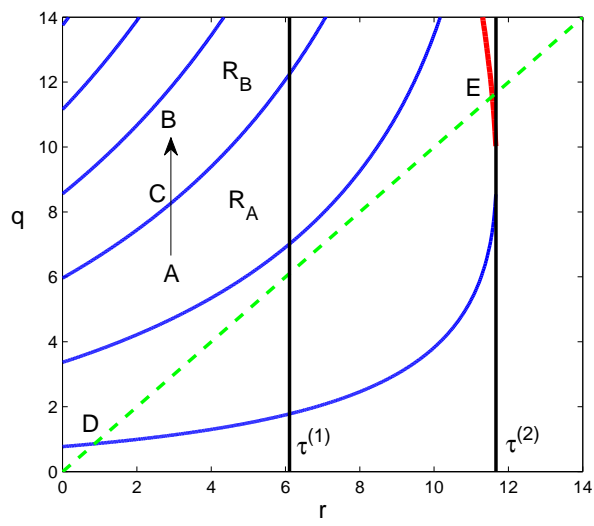

Fig. 2. The critical delay curves of the population model (1)

In Fig. 2 the blue and red curves are the critical delay curves of the population model (1). The blue ones correspond to the graphs of a family of functions $\tau_{k}^{(i)}(\tau)+2 l \pi / \omega_{k}^{(i)}(\tau)$ parameterized by integer $l$ and $i=1,2, k=1$. The red curves corresponds to the graphs of a family of functions $\tau_{2}^{(2)}(\tau)+2 l \pi / \omega_{2}^{(2)}(\tau)$ parameterized by the integer $l$. Now consider two points $\mathrm{A}$ and $\mathrm{B}$, which forms a vertical line segment. $A, B$ are in two different but adjacent parameter regions denoted by $R_{A}, R_{B}$ respectively. Let the parameter $(r, q)$ start moving from $A$ vertically towards $B$, or in other words we fix $r$ and increase $q$ continuously. When the point reaches a boundary curve at Point $C$ some imaginary roots appear. By monitoring whether this imaginary root move towards R.H.P or L.H.P as $\tau$ further increases one can determine how the the number of unstable roots changes as the parameter crosses the boundary curve and enters $R_{B}$ from $R_{A}$. The next result from [18] shows that the moving direction of the imaginary roots are captured by the differential information of Function $F$ with the parameter $(r, q)$ fixed at the crossing point.

Lemma 1: Let $r$ in (18) be fixed. Suppose $\lambda=j \omega^{*}$ is an unrepeated root of (18) for $q=q^{*}$. Then for $(\lambda, q)$ in a neighbourhood of $\left(j \omega^{*}, q^{*}\right),(18)$ defines $\lambda$ as a differentiable function of $q$ denoted by $\lambda(q)$. The following holds:

$$
\operatorname{sgn}\left(\Re\left(\frac{d \lambda\left(q^{*}\right)}{d q}\right)\right)=\operatorname{sgn}\left(\partial_{\omega} F\left(\omega^{*}, r\right)\right) .
$$

The original system (2) is just equivalent to (18) with restriction $r=q=\tau$. Therefore as the delay value in (2) sweeps through $\mathscr{I}$, on the $r-q$ plane the parameter point $(r, q)$ moves along the 45 degree dashed green line in Fig. 2 and thus enters or leaves different stability regions of the $r-q$ plane. The dashed green line intersects the critical delay curves at Point $D$ and $E$, therefore that at these points imaginary roots appear. This conclusion is consistent with our previous analysis based on (12). Then the crossing direction of these imaginary roots may be determined by applying Lemma 1 and taking into account whether the 45 degree line crosses the critical delay curves from below or from above. In the next section, we will exploit this geometric idea to carry out the stability analysis.

\section{B. Crossing conditions for the original system}

In this section we study the crossing direction of the imaginary roots of (2) by considering (18) with the restriction $r=q=\tau$. We need some notation for the formulation of the main theorem.

To any number $r \in\left(\tau^{(i-1)}, \tau^{(i)}\right]$ we assign a fixed number $r_{-} \in\left(\tau^{(i-1)}, r\right)$ requiring that $D_{\tau}(\lambda)$ admits no imaginary root for $\tau \in\left[r_{-}, r\right)$. We also assign a a fixed numbers $r_{+} \in$ $\left(r, \tau^{(i)}\right)$ to any $r \in\left[\tau^{(i-1)}, \tau^{(i)}\right)$ requiring that $D_{\tau}(\lambda)$ admits no imaginary root for $\tau \in\left(r, r_{+}\right]$.

Suppose $\tau^{*} \in\left[\tau^{(i-1)}, \tau^{(i)}\right)$. Let $\lambda=j \omega^{*}, \omega^{*}>0$ be a characteristic root of $D_{\tau^{*}}(\lambda)$. If $\tau^{*}=\tau^{(i-1)}$, set $i^{\prime}=i-1$, otherwise set $i^{\prime}=i$. Introduce a set $\mathscr{K}_{+}\left(\tau^{*}\right)$ which collects each $k$ that satisfies the following two conditions simultaneously:

$$
\begin{gathered}
\theta_{k}^{(i)}\left(\tau^{*}\right)=2 l \pi, l \text { integer, } \\
\lim _{\tau \rightarrow \tau^{*+}} \operatorname{sgn}\left(\theta_{k}^{(i)}(\tau)-\theta_{k}^{(i)}\left(\tau^{*}\right)\right)=-1 .
\end{gathered}
$$

Similarly, if $\tau^{*} \neq \tau^{l}$ define $\mathscr{K}_{-}\left(\tau^{*}\right)$ as the set of each $k^{\prime}$ that satisfies the following two conditions:

$$
\begin{gathered}
\theta_{k^{\prime}}^{\left(i^{\prime}\right)}\left(\tau^{*}\right)=2 l \pi, l \text { integer, } \\
\lim _{\tau \rightarrow \tau^{*-}} \operatorname{sgn}\left(\theta_{k^{\prime}}^{\left(i^{\prime}\right)}(\tau)-\theta_{k^{\prime}}^{\left(i^{\prime}\right)}\left(\tau^{*}\right)\right)=-1 .
\end{gathered}
$$

We note that (24) is just equivalent to

$$
\lim _{r \rightarrow \tau^{*+}} \operatorname{sgn}\left(r-\tau_{k}^{(i)}(r)-\theta_{k}^{(i)}\left(\tau^{*}\right) / \omega_{k}^{(i)}(r)\right)=-1
$$


and (26) is equivalent to

$$
\lim _{r \rightarrow \tau^{*-}} \operatorname{sgn}\left(r-\tau_{k^{\prime}}^{\left(i^{\prime}\right)}(r)-\theta_{k^{\prime}}^{\left(i^{\prime}\right)}\left(\tau^{*}\right) / \omega_{k^{\prime}}^{\left(i^{\prime}\right)}(r)\right)=-1
$$

Therefore $\mathscr{K}_{+}\left(\tau^{*}\right)$ is just the set that collects all index $k$ such that on the $r-q$ plane some branches of the critical delay curves of the form $\tau_{k}^{(i)}(r)+\theta_{k}^{(i)}\left(\tau^{*}\right) / \omega_{k}^{(i)}(r)$ that approaches the 45 degree line $q=r$ from below as $r \rightarrow \tau^{*+}$ and reaches the point $\left(\tau^{*}, \tau^{*}\right)$ at $r=\tau^{*}$. We have the same interpretation for the set $\mathscr{K}_{-}\left(\tau^{*}\right)$ except that we should replace ' $r \rightarrow \tau^{*+}$, with ' $r \rightarrow \tau^{*-}$, and $i$ with $i^{\prime}$.

We further decompose each set $\mathscr{K}_{+}\left(\tau^{*}\right)$ or $\mathscr{K}_{-}\left(\tau^{*}\right)$ into subsets by the frequency of different imaginary eigenvalues. Define

$$
\begin{aligned}
& \mathscr{K}_{+}\left(\omega^{*}, \tau^{*}\right)=\left\{k \in \mathscr{K}_{+}\left(\tau^{*}\right) \mid \omega_{k}^{(i)}\left(\tau^{*}\right)=\omega^{*}\right\}, \\
& \mathscr{K}_{-}\left(\omega^{*}, \tau^{*}\right)=\left\{k \in \mathscr{K}_{-}\left(\tau^{*}\right) \mid \omega_{k}^{\left(i^{\prime}\right)}\left(\tau^{*}\right)=\omega^{*}\right\} .
\end{aligned}
$$

Return to the population model (1), consider Point $D=\left(\tau_{1}, \tau_{1}\right)$ in the second plot of Fig.1. Since at this point $\Omega_{F}$ contains only one element, namely $\omega_{1}^{(1)}\left(\tau_{1}\right)$ and at Point $D$ the curve of $\theta_{1}^{(1)}(\tau)$ has a tangent with positive slope, we deduce that $\mathscr{K}_{-}\left(\tau_{1}\right)=\mathscr{K}_{-}\left(\omega_{1}^{(1)}\left(\tau_{1}\right), \tau_{1}\right)=\{1\}$ and $\mathscr{K}_{+}\left(\tau_{1}\right)=\mathscr{K}_{+}\left(\omega_{1}^{(1)}\left(\tau_{1}\right), \tau_{1}\right)=\{\phi\}$.

Theorem 1: Suppose $\tau^{*} \in\left[\tau^{(i-1)}, r^{(i)}\right)-\left\{\tau^{l}\right\}$ and $\lambda=$ $j \omega^{*}, \omega^{*}>0$ is a characteristic root of $D_{\tau^{*}}(\lambda)$. Let $i^{\prime}=i-1$ if $\tau^{*}=\tau^{(i-1)}$, otherwise let $i^{\prime}=i$. The following holds

$$
\operatorname{Inc}\left(\omega^{*}, \tau^{*}\right)=\sum_{k \in \mathscr{K}_{+}\left(\omega^{*}, \tau^{*}\right)} \operatorname{sgn}_{k}^{(i)}-\sum_{k \in \mathscr{K}_{-}\left(\omega^{*}, \tau^{*}\right)} \operatorname{sgn}_{k}^{\left(i^{\prime}\right)}
$$

where the quantity $\operatorname{sgn}_{k}^{(i)}$ is defined in (10).

Due to the limited space, here we only sketch the proof of Theorem 1, which is based on the geometric construction illustrated in Fig.3. On the $r-q$ parameter plane, we define four points whose coordinates are given as follows

$$
\begin{aligned}
& A=\left(\tau^{*}-\varepsilon_{1}, \tau^{*}-\varepsilon_{1}\right), B=\left(\tau^{*}-\varepsilon_{1}, \tau^{*}-\varepsilon_{0}\right), \\
& C=\left(\tau^{*}+\varepsilon_{1}, \tau^{*}-\varepsilon_{0}\right), D=\left(\tau^{*}+\varepsilon_{1}, \tau^{*}+\varepsilon_{1}\right),
\end{aligned}
$$

where $\varepsilon_{1}, \varepsilon_{0}$ are two small positive numbers with $\varepsilon_{0}>\varepsilon_{1}$ and are chosen in such a way that the following two conditions hold:

(i) Segment $A B$ intersects the graphs of $\tau_{k}^{\left(i^{\prime}\right)}(\tau)+$ $\theta_{k}^{\left(i^{\prime}\right)}\left(\tau^{*}\right) / \omega_{k}^{\left(i^{\prime}\right)}(\tau)$ if and only if $k \in \mathscr{K}_{-}\left(\tau^{*}\right)$. (ii) Segment $C D$ intersects the graphs of $\tau_{k}^{(i)}(\tau)+\theta_{k}^{(i)}\left(\tau^{*}\right) / \omega_{k}^{(i)}(\tau)$ if and only if $k \in \mathscr{K}_{+}\left(\tau^{*}\right)$.

Denote the intersections between Segment $A B$ and the the graphs of $\tau_{k}^{\left(i^{\prime}\right)}(\tau)+\theta_{k}^{\left(i^{\prime}\right)}\left(\tau^{*}\right) / \omega_{k}^{\left(i^{\prime}\right)}(\tau), k \in \mathscr{K}_{-}\left(\omega^{*}, \tau^{*}\right)$ as $P_{1}, P_{2}, \cdots P_{K-}$. Denote as well the intersections between Segment $C D$ and the the graphs of $\tau_{k}^{(i)}(\tau)+\theta_{k}^{(i)}\left(\tau^{*}\right) / \omega_{k}^{\left(i^{\prime}\right)}(\tau)$, $k \in \mathscr{K}_{+}\left(\omega^{*}, \tau^{*}\right)$ as $Q_{1}, Q_{2}, \cdots Q_{K+}$.

Let $\mathscr{B}_{\delta}\left(j \omega^{*}\right)$ be a ball on the complex plane centred at $j \omega^{*}$ with radius $\delta$. Let $\mu^{*}$ be the multiplicity of the characteristic root $j \omega^{*}$ of $D_{\tau^{*}}(\lambda)$. For any sufficiently small $\delta$, we can make $\varepsilon_{0}, \varepsilon_{1}$ sufficiently small and thus ensure there are exactly $\mu^{*}$ characteristic roots of $D_{r q}(\lambda)$ in $\mathscr{B}_{\delta}\left(j \omega^{*}\right)$ as $(r, q)$ moves along the path $A B C D$. Consequently we can deduce that as $(r, q)$ moves alone the path $A B C D$, a unique imaginary characteristic root appears inside $\mathscr{B}_{\delta}\left(j \omega^{*}\right)$ if and only if $(r, q)$ passes through $P_{1}, P_{2}, \cdots, P_{K-}$ and $Q_{1}, Q_{2}, \cdots, Q_{K+}$. By applying Lemma 1 at each of these points to determine the crossing directions, (27) can be concluded.

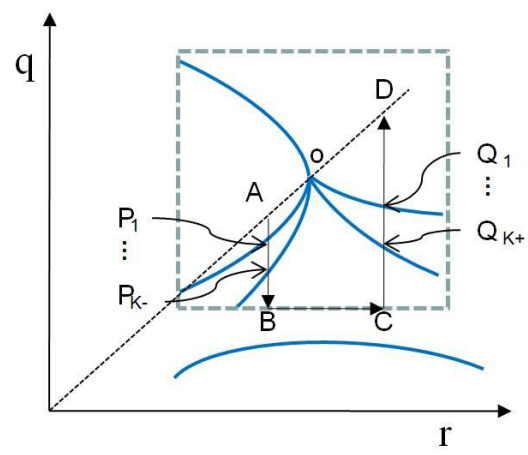

Fig. 3. Illustration for the proof of Theorem 1. The blue curves represents the critical delay curves, namely the graph of $\tau_{k}^{(i)}(r)+\theta_{k}^{(i)}\left(\tau^{*}\right) / \omega_{k}^{(i)}(r)$ for some appropriate indices $i, k$.

Corollary 1: Suppose $\tau^{*} \in\left[\tau^{(i-1)}, r^{(i)}\right)-\left\{\tau^{l}\right\}$ is a critical delay. Let $i^{\prime}=i-1$ if $\tau^{*}=\tau^{(i-1)}$, otherwise let $i^{\prime}=i$. The following holds

$$
\operatorname{Inc}\left(\tau^{*}\right)=\sum_{k \in \mathscr{K}_{+}\left(\tau^{*}\right)} 2 \operatorname{sgn}_{k}^{(i)}-\sum_{k \in \mathscr{K}_{-}\left(\tau^{*}\right)} 2 \operatorname{sgn}_{k}^{\left(i^{\prime}\right)}
$$

This corollary is a direct consequence of Theorem 1 and (16). The following results can be derived from Theorem 1. We omit the proof.

Proposition 1: Suppose $\tau^{*} \in\left[\tau^{(i-1)}, \tau^{(i)}\right)-\left\{\tau^{l}\right\}$, and $\lambda=$ $j \omega_{k}^{(i)}\left(\tau^{*}\right)$ is an unrepeated characteristic root of $D_{\tau^{*}}(\lambda)$. Denote $\omega^{*}=\omega_{k}^{(i)}\left(\tau^{*}\right)$. For $\tau$ in a neighbourhood of $\tau^{*}$ this root is a function of $\tau$ written as $\lambda(\tau)$. If $\tau^{*}=\tau^{(i-1)}$, set $i^{\prime}=i-1$, otherwise let $i^{\prime}=i$. Let $k^{\prime}$ be the index that satisfies (25). We have the following criterion concerning the crossing direction of the imaginary root $\lambda\left(\tau^{*}\right)$ :

$$
\left.\lim _{\varepsilon \rightarrow 0^{+}} \operatorname{sgn}(\Re(\lambda(\tau)))\right|_{\tau=\tau^{*}-\varepsilon} ^{\tau=\tau^{*}+\varepsilon}=2 \operatorname{Inc}\left(\omega^{*}, \tau^{*}\right),
$$

$$
\begin{aligned}
\operatorname{Inc}\left(\omega^{*}, \tau^{*}\right)= & \frac{1}{2} \operatorname{sgn}_{k}^{(i)} \times\left(\operatorname{sgn}\left(\theta_{k}^{(i)}\left(\tau_{+}^{*}\right)-\theta_{k}^{(i)}\left(\tau^{*}\right)\right)\right. \\
& \left.-\operatorname{sgn}\left(\theta_{k^{\prime}}^{\left(i^{\prime}\right)}\left(\tau_{-}^{*}\right)-\theta_{k^{\prime}}^{\left(i^{\prime}\right)}\left(\tau^{*}\right)\right)\right) .
\end{aligned}
$$

In the last proposition, if $\tau^{*} \neq \tau^{(i-1)}$, we must have $i=i^{\prime}$ and $k=k^{\prime}$, then (30) clearly shows that the crossing direction of the imaginary root $j \omega^{*}$ associated with the frequency function $\omega_{k}^{(i)}(\tau)$ is determined by the monotonicity of the phase function $\theta_{k}^{(i)}(\tau)$ at $\tau^{*}$ as well as the quantity $\operatorname{sgn}_{k}^{(i)}$. On the other hand, if in the last proposition $\tau^{*}=\tau^{(i-1)}$, it is easy to see $\theta_{k}^{(i)}\left(\tau^{*}\right)=\theta_{k^{\prime}}^{\left(i^{\prime}\right)}\left(\tau^{*}\right)+2 l \pi$, for some integer $l$. 
Therefore one can concatenate $\theta_{k}^{(i)}(\tau)$ with $\theta_{k^{\prime}}^{\left(i^{\prime}\right)}(\tau)+2 l \pi$ at $\tau^{*}$ to form one continuous phase function, say $\theta(\tau)$. Then from (30) we can deduce the same correlation between the crossing direction of the imaginary characteristic root $j \omega^{*}$ and the monotonicity of $\theta(\tau)$ at $\tau^{*}$ as in the case of $i=i^{\prime}$.

Proposition 2: Suppose $\tau^{*} \in\left[\tau^{(i-1)}, \tau^{(i)}\right)$ and $j \omega_{k}^{(i)}\left(\tau^{*}\right)=$ $j \omega^{*}$ is an unrepeated imaginary characteristic root of $D_{\tau^{*}}(\lambda)$. In a neighborhood of $\left(j \omega^{*}, \tau^{*}\right)$, the characteristic equation (2) implicitly determines $\lambda$ as a function of $\tau$ denoted as $\lambda(\tau)$. It follows that

$$
\lim _{\tau \rightarrow \tau^{*+}} \operatorname{sgn}(\Re(\lambda(\tau)))=\operatorname{sgn}\left(\theta_{k}^{(i)}\left(\tau_{+}^{*}\right)-\theta_{k}^{(i)}\left(\tau^{*}\right)\right) \operatorname{sgn}_{k}^{(i)} .
$$

Assume that there exists a positive integer $n_{d}$ such that $\left(\frac{d}{d \tau}\right)^{n_{d}} P(j \omega, \tau)$ and $\left(\frac{d}{d \tau}\right)^{n_{d}} Q(j \omega, \tau)$ exist at $\left(j \omega^{*}, \tau^{*}\right)$. Furthermore, assume that $n_{d}$ satisfies $\left(\frac{d}{d \tau}\right)^{l} \theta_{k}^{(i)}\left(\tau^{*}\right)=0$, for $1 \leq l<n_{d}$ and $\left(\frac{d}{d \tau}\right)^{n_{d}} \boldsymbol{\theta}_{k}^{(i)}\left(\tau^{*}\right) \neq 0$. Then the following holds:

$$
\begin{aligned}
\operatorname{sgn}\left(\left(\frac{d}{d \tau}\right)^{l} \Re\left(\lambda\left(\tau^{*}\right)\right)\right)= & \operatorname{sgn}\left(\left(\frac{d}{d \tau}\right)^{l} \theta_{k}^{(i)}\left(\tau^{*}\right)\right) \\
& \times \operatorname{sgn}_{k}^{(i)},
\end{aligned}
$$

for $1 \leq l \leq n_{d}$.

It is worth mentioning that Proposition 2 is reduced to the first-order root-crossing criteria given in [17] when $l=1$ in (32).

\section{Stability of the population model}

In the previous analysis of the population model (1) we have already decomposed $\mathscr{I}$ into $\mathscr{I}^{(i)}, i=1,2,3$ and we know $\tau^{(0)}=0, \tau^{(1)} \approx 6.108, r^{(2)} \approx 11.695$, and $\tau^{(3)}=\tau^{u}=$ 14. We also have $\mathscr{T}_{c}=\left\{\tau_{1}, \tau_{2}\right\}$ and $\tau_{1} \approx 0.845, \tau_{2} \approx 11.566$. It is easy to check $N^{u}(0)=0$. We pick arbitrarily a number in $\left(\tau^{(1)}, \tau_{1}\right)$ as $\tau_{1-}$. and a number in $\left(\tau_{1}, \tau^{(2)}\right)$. From the lower diagram of Fig. 1 it is easy to $\operatorname{see} \operatorname{sgn}\left(\theta_{1}^{(1)}\left(\tau_{1-}\right)-\right.$ $\left.\theta_{1}^{(1)}\left(\tau_{1}\right)\right)=-1$ and $\operatorname{sgn}\left(\theta_{1}^{(1)}\left(\tau_{1+}\right)-\theta_{1}^{(1)}\left(\tau_{1}\right)\right)=1$. Simple computation shows $\operatorname{sgn}_{1}^{(1)}=1$. It thus follows from (30) that $\operatorname{Inc}\left(\tau_{1}\right)=2 \operatorname{Inc}\left(j \omega_{1}^{(1)}\left(\tau_{1}^{*}\right), \tau_{1}\right)=2$. In other words, the pair of characteristic roots $\pm j \omega_{1}^{(1)}\left(\tau_{1}\right)$ crosses the imagine axis towards R.H.P as $\tau$ increases beyond $\tau_{1}$. We can make the same conclusion about the crossing direction of this pair of imaginary root by invoking Proposition 2 and noticing $\frac{d}{d \tau} \theta_{1}^{(1)}\left(\tau_{1}\right)>0$. We now consider the critical delay $\tau_{2}$. We pick arbitrarily two numbers $\tau_{2-} \in\left(\tau^{(1)}, \tau_{2}\right)$ and $\tau_{2+} \in$ $\left(\tau_{2}, \tau^{(2)}\right)$. From the lower diagram of Fig. 1 it is easy to see $\operatorname{sgn}\left(\theta_{2}^{(2)}\left(\tau_{2-}\right)-\theta_{2}^{(2)}\left(\tau_{2}\right)\right)=-1, \operatorname{sgn}\left(\theta_{2}^{(2)}\left(\tau_{2+}\right)-\theta_{2}^{(2)}\left(\tau_{2}\right)\right)=$ 1 . We can verify $\operatorname{sgn}_{2}^{(2)}=-1$. Applying (30) we deduce $\operatorname{Inc}\left(\tau_{2}\right)=2 \operatorname{Inc}\left(j \omega_{2}^{(2)}\left(\tau_{2}\right), \tau_{2}\right)=-2$. Therefore the pair of roots $\pm j \omega_{2}^{(2)}\left(\tau_{2}\right)$ cross the imaginary axis towards L.H.P as $\tau$ increases and sweeps through $\tau_{2}$. It is easy to verify $\frac{d}{d \tau} \theta_{2}^{(2)}\left(\tau_{2}\right)>0$, then Proposition 2 yields the same result. Consequently, we conclude that the system is asymptotically stable for $\tau \in\left[0, \tau_{1}\right) \cup\left(\tau_{2}, 14\right]$ and has two unstable characteristic roots for $\tau \in\left(\tau_{1}, \tau_{2}\right)$.

\section{Conclusion}

In this paper stability of time-delay systems with delaydependent coefficients is studied. A systematic method is proposed to determine the number of unstable characteristic roots for a given delay interval of interest. The delay interval is decomposed into subintervals such that within each subinterval a fixed number of frequency functions and phase functions are well defined. Using these functions we present various criteria concerning the crossing direction of the imaginary characteristic roots. It is shown that the crossing direction of these roots is closely related to the monotonicity of the phase functions at the critical delays. In comparison with the previous related work, our analysis is based on relaxed assumptions and yields more general results applicable also to multiple imaginary roots. The twoparameter perspective provides geometric insight into our problem and allows for more intuitive interpretation of the results.

\section{REFERENCES}

[1] K. L. Cooke and P. van den Driessche, "On zeroes of some transcendental equations," Funkcialaj Ekvacioj, vol. 29, pp. 77-90, 1986.

[2] F. G. Boese, "Stability with respect to the delay: On a paper by K.L. Cooke and P. van den Driessche," J. Math. Anal. Appl, vol. 228, pp. 293-321, 1998

[3] Gu, K., Kharitonov, V. L., \&Chen, J. (2003). Stability of time-delay systems.

[4] Gu, K. (2012). A review of some subtleties of practical relevance for time-delay systems of neutral type. ISRN Applied Mathematics, Vol 2012, Article ID 725783, 46 pages, doi: 10.5402/2012/725783.

[5] Michiels, W., \& Niculescu, S. I. (2014). Stability, Control, and Computation for Time-Delay Systems: An Eigenvalue-Based Approach (Vol. 27). Siam.

[6] S. I. Niculescu : Delay effects on stability. A robust control approach, Springer: Heidelberg, Series: LNCIS, vol. 269, 2001.

[7] P. Fu, J. Chen, S. I. Niculescu. "High-order analysis of critical stability properties of linear time-delay systems." In American Control Conference, 2007. ACC'07, pp. 4921-4926.

[8] Chen, J., Gu, G., Carl N. Nett, A new method for computing delay margins for stability of linear delay systems, Systems \& Control Letters, Volume 26, Issue 2, 22 September 1995, Pages 107-117.

[9] Beretta, E., Kuang, Y. (2002). Geometric stability switch criteria in delay differential systems with delay dependent parameters. SIAM Journal on Mathematical Analysis, 33(5), 1144-1165.

[10] R. M. Nisbet, W. S. C. Gurney, J. A. J. Metz, Stage structure models applied in evolutionary ecology, Biomathematics, 18 (1989), pp. 428449.

[11] Wilmot-Smith, A. L., et al. "A time delay model for solar and stellar dynamos." The Astrophysical Journal 652.1 (2006): 696.

[12] F. Crauste, "Global Asymptotic Stability and Hopf Bifurcation for a Blood Cell Production Model." on-line document (2005).

[13] K. Walton and J. E. Marshall, "Direct method for TDS stability analysis," IEE Proc. vol. 134, part D, pp. 101-107, 1987.

[14] E. N. Gryazina, B. T. Polyak, and A. A. Tremba, "D-decomposition technique state-of-the-art," Automation and Remote Control, vol. 69, no. 12, pp. 1991-2026, 2008.

[15] L. E. El'Sgol'ts and S. B. Norkin, Introduction to the Theory and Application of Differential Equations with Deviating Arguments, Translated by J. L. Casti, Academic Press, New York, 1973.

[16] M. S. Lee and C. S. Hsu, "On the $\tau$-decomposition method of stability analysis for retarded dynamical systems," SIAM J. Control, 7:249, 259, 1969.

[17] K. Gu, C. Jin, I. Boussaada and S. I. Niculescu, "Towards more general stability analysis of systems with delay-dependent coefficients," 2016 IEEE 55th Conference on Decision and Control (CDC), Las Vegas, NV, 2016, pp. 3161-3166.

[18] K.L. Cooke, Pauline Van Den Driessche. "On zeroes of some transcendental equations.” Funkcialaj Ekvacioj 29.1 (1986): 77-90. 